\title{
Spin Diffusion in Double-Exchange Manganites
}

\author{
A. L. Chernyshev ${ }^{1,2}$ and R. S. Fishman ${ }^{2}$ \\ ${ }^{1}$ Department of Physics and Astronomy, University of California, Irvine CA 92697 \\ ${ }^{2}$ Condensed Matter Sciences Division, Oak Ridge National Laboratory, P.O. Box 2008, Oak Ridge, Tennessee 37831
}

(Dated: November 21, 2018)

\begin{abstract}
The theoretical study of spin diffusion in double-exchange magnets by means of dynamical meanfield theory is presented. We demonstrate that the spin-diffusion coefficient becomes independent of the Hund's coupling $J_{H}$ in the range of parameters $J_{H} S \gg W \gg T, W$ being the bandwidth, relevant to colossal magnetoresistive manganites in the metallic part of their phase diagram. Our study reveals a close correspondence as well as some counterintuitive differences between the results on Bethe and hypercubic lattices. Our results are in accord with neutron scattering data and with previous theoretical work for high temperatures.
\end{abstract}

PACS numbers: 72.10.-d, 72.15.Lh, 75.30.Vh, 75.40Gb

Spin diffusion dominates the low- $\omega$, low-k excitation spectrum of a magnet in its paramagnetic state and contains important information about the spin dynamics. In the past, spin diffusion was studied intensively in Heisenberg systems and, recently, has been investigated both theoretically and experimentally for strongly-correlated itinerant magnets 1.2 .3 The current growth of interest in spintronics requires understanding how local spins relax through their interactions mediated by itinerant charge carriers rather than through their direct interactions with each other $\underline{\underline{4}}$ Among such systems are the colossal magnetoresistive (CMR) manganites which are ferromagnetic metals in a large part of their phase diagram $\frac{\underline{\underline{5}}}{\text { Recent sys- }}$ tematic neutron scattering experiments on the ferromagnetic CMR materials revealed a peak centered at $\omega=0$ associated with the spin diffusion. This peak was also seen below the ordering temperature indicating electronic inhomogeneity with regions having lower $T_{c}$ 's.

In this Letter we present a comprehensive, selfconsistent, microscopic calculation of spin diffusion applying dynamical mean-field theory (DMFT) to the double-exchange (DE) model. We demonstrate that the spin-diffusion coefficient $\mathcal{D}_{s}$ is related to the local singleparticle Green's function and can be evaluated as a function of doping and temperature. Our results agree quantitatively with neutron scattering data on manganites for a range of doping concentrations. Thus, our approach creates a framework for the self-consistent study of diffusive spin dynamics in many real materials, including magnetically doped semiconductors.

Following the general hydrodynamic arguments of Ref. $\underline{6}$, we write the generalized susceptibility of a paramagnet for low energies and long wavelengths as

$$
\chi(\mathbf{q}, \omega) \simeq \chi(\mathbf{q}) \frac{\mathcal{D}_{s} q^{2}}{-i \omega+\mathcal{D}_{s} q^{2}},
$$

which through the fluctuation-dissipation theorem gives the neutron-scattering dynamical structure factor $\mathcal{S}(\mathbf{q}, \omega) \simeq 2\left[n_{B}(\omega)+1\right] \operatorname{Im} \chi(\mathbf{q}, \omega)$, where $\chi(\mathbf{q})$ is the static susceptibility at wave-vector $\mathbf{q}$ and $n_{B}(\omega)=\left[e^{\omega / T}-1\right]^{-1}$. We take $\hbar=k_{B}=1$ throughout this paper. Further, the generalized susceptibility can be related to the spin current-current correlation function using the dispersion relations ${ }^{\underline{T}}$ and the continuity equation $\partial S^{\alpha}(\mathbf{r}, t) / \partial t=$ $-\nabla_{i} j_{i}^{\alpha}(\mathbf{r}, t)$ :

$$
\chi(\mathbf{q}, \omega)=-\frac{q^{2} a^{2}}{\omega^{2}}[\Pi(\mathbf{q}, \omega)-\Pi(\mathbf{q}, 0)],
$$

where $\Pi_{i j}^{\alpha \beta}(\mathbf{q}, \omega)=-i \int d t e^{i \omega t} \theta(t)\left\langle\left[j_{i}^{\alpha \dagger}(\mathbf{q}, t), j_{j}^{\beta}(\mathbf{q}, 0)\right]\right\rangle$ is the retarded current-current correlation function ${ }^{8}$, $j_{i}^{\alpha}(\mathbf{q}, t)$ is the $i$ th component of the spin current for the $\alpha$-spin projection, $i=1 \ldots d, d$ is the dimensionality, and $a$ is the lattice constant. We use the isotropy of the spins above $T_{c}$ and assume the isotropy of real space to suppress the indices in $\Pi_{i j}^{\alpha \beta}(\mathbf{q}, \omega)=\Pi(\mathbf{q}, \omega) \delta_{\alpha \beta} \delta_{i j}$. Combining Eqs. (1) and (2) in the $\mathbf{q}, \omega \rightarrow 0$ limit, we write the Einstein relation between the spin-diffusion coefficient and the spin conductivity $\sigma_{s}$ (which in general is distinct from the particle conductivity) as

$$
\mathcal{D}_{s} \chi=\sigma_{s}=-a^{2} \lim _{\omega \rightarrow 0} \frac{\operatorname{Im}[\Pi(0, \omega)]}{\omega},
$$

where $\chi=\chi(\mathbf{q}=0)$. These expressions are general and do not depend on the microscopic model.

We now consider the DE model with Hamiltonian

$$
\mathcal{H}=-t \sum_{\langle i j\rangle \sigma}\left(c_{i \sigma}^{\dagger} c_{j \sigma}+\text { H.c. }\right)-2 J_{H} \sum_{i} \mathbf{S}_{i} \cdot \mathbf{s}_{i},
$$

where $t$ is the nearest-neighbor kinetic energy, $J_{H}$ is the Hund's coupling between the local $\mathrm{Mn}^{3+} S=3 / 2$ spin and the electronic spin $\mathbf{s}=c_{\gamma}^{\dagger} \hat{\boldsymbol{\sigma}}_{\gamma \delta} c_{\delta} / 2$, and $\hat{\boldsymbol{\sigma}}$ are the Pauli matrices. To describe the multitude of phases in manganites requires that the orbital, phonon, or JahnTeller terms be included in the above model ${ }^{9}$ However, the magnetic properties of these materials in the metallic part of their phase diagram, such as the magnetic excitation spectrum and the ferromagnetic transition temperature,, 10.11 .12 are quantitatively well described by the model in Eq. (4). Therefore, such a model must be also capable of describing spin diffusion in these systems $\stackrel{13}{n}$ Regardless of modifications of the DE model 
needed to describe a particular real system, elucidating the dynamic properties of this basic model of stronglycorrelated itinerant magnets is an important task on its own.

Within the DE model there is no direct interaction between the local spins. Thus, the total on-site spin $\mathbf{S}_{l}^{t o t}=\mathbf{S}_{l}+\mathbf{s}_{l}$ commutes with the exchange part of Eq. (4) and the spin current can be expressed in terms of electronic operators only $\stackrel{14}{14}_{i}^{\alpha}(\mathbf{q})=\sum_{\mathbf{k}} v_{\mathbf{k}}^{i} c_{\mathbf{k}, \gamma}^{\dagger} \hat{\sigma}_{\gamma \delta}^{\alpha} c_{\mathbf{k}-\mathbf{q}, \delta} / 2$, where $v_{\mathbf{k}}^{i}=\nabla^{i} \varepsilon_{\mathbf{k}}$ and $\varepsilon_{\mathbf{k}}=-2 t \sum_{i=1}^{d} \cos k_{i} a$. This is simply another way of saying that electrons mediate the magnetic relaxation processes in an itinerant system.

The physical situation relevant to manganites corresponds to strong Hund's coupling $J_{H} S \gg W$. Since the characteristic relaxation time for the electronic spin is short and the spin relaxation is essentially local, perturbative approaches to the spin diffusion ${ }^{15}$ are inapplicable. Therefore, we employ DMFT, which takes into account the local dynamics in strongly-correlated systems and has been successfully applied to a number of problems $\frac{16.17}{16}$ Using DMFT also simplifies our problem significantly because the higher-order diagrams in the current-current correlation function of Eq. (3), often referred to as vertex corrections, are identically zero within this approach $\underline{\underline{16}}$

Thus, to evaluate the spin-diffusion coefficient we apply the standard Matsubara formalism to Eq. (3) using the above definition of the spin current:

$$
\frac{\mathcal{D}_{s} \chi}{a^{2}}=\frac{\pi}{2} \sum_{\mathbf{k}}\left(v_{\mathbf{k}}^{i}\right)^{2} \int_{-\infty}^{\infty} d \nu A_{\mathbf{k}}(\nu)^{2}\left(-\left.\frac{\partial n(x)}{\partial x}\right|_{x=\nu}\right),
$$

where $\mathbf{k}$ and $\nu$ are the internal momentum and frequency of the "bubble" diagram, respectively. Here $v_{\mathbf{k}}^{i}=2 t \sin k_{i} a, A_{\mathbf{k}}(\nu)=-(1 / \pi) \operatorname{Im} G_{\mathbf{k}}(\nu)$ is the electronic spectral function, $n(\nu)=\left[e^{(\nu-\mu) / T}+1\right]^{-1}$ is the Fermi function, and $\mu$ is the chemical potential.

The DMFT imposes a special form of the Green's function in which the self-energy is k-independent and is defined from a self-consistency condition specified below. Within the DMFT parameters of the model are rescaled such that $\bar{t}=t \sqrt{z}$ is finite as the dimensionality $d \rightarrow \infty$, where $z=2 d$ is the number of nearest neighbors ${ }^{16} \mathrm{In}$ the following $\sqrt{2} \bar{t}$ is set to unity. Generally, the $d=\infty$ limit is well defined for Bethe and hypercubic lattice geometries. While the semicircular electronic density of states (DOS) of the Bethe lattice is convenient for calculations, the Bethe lattice itself lacks the translational invariance and inversion symmetry implicitly used to obtain Eq. (5). Thus, it is important to determine whether the results on the Bethe lattice are equivalent to the results on the hypercubic lattice, which is free from such deficiencies. Since many problems have been studied using the Bethe lattice, $\stackrel{16}{\longleftarrow}$ this comparison will have an even broader significance for the DMFT in general.

We briefly sketch here the DMFT equations for the DE model $\stackrel{10.18}{=}$ Since the self-energy is local, one can change $G_{\mathbf{k}}(E) \Rightarrow G_{\varepsilon}(E)=[E-\varepsilon-\Sigma(E)]^{-1}$, and $\sum_{\mathbf{k}} \Rightarrow \int d \varepsilon \rho_{0}(\varepsilon)$, where $\varepsilon=\varepsilon_{\mathbf{k}}$, and $\rho_{0}^{B}(\varepsilon)=\sqrt{2-\varepsilon^{2}} / \pi$ and $\rho_{0}^{H}(\varepsilon)=\exp \left(-\varepsilon^{2}\right) / \sqrt{\pi}$ are the bare DOS's for Bethe and hypercubic lattices, respectively. The properties of the system are obtained from the local Green's function:

$$
g(E)=\int d \varepsilon \frac{\rho_{0}(\varepsilon)}{E-\varepsilon-\Sigma(E)}
$$

where the self-energy is defined from $\Sigma(E)=g_{0}^{-1}(E)-$ $g^{-1}(E)$, reminiscent of the Dyson equation, where $g_{0}^{-1}(E)$ is the "Weiss" function containing the dynamic influence of the environment on a given local site. The solution of the single-site problem provides a relation between $g_{0}(E)$ and $\Sigma(E) \stackrel{10.16}{1}$ In the paramagnetic state and in the quasiclassical limit $S \gg 1$, such a relation is particularly simple $\mathbb{1 0}^{10} \Sigma(E)=\left(J_{H} S\right)^{2} g_{0}(E)$, which yields

$$
g(E)=\frac{\Sigma(E)}{\left(J_{H} S\right)^{2}-\Sigma(E)^{2}} .
$$

Together with Eq. (6), this gives a self-consistent condition for $\Sigma(E)$ or $g(E)$.

We now compare the Bethe and hypercubic solution for the single-particle properties. Fig. 1 shows the evolution of the interacting DOS $N(\omega)=-(1 / \pi) \operatorname{Im} g(\omega)$ for several values of $J_{H} S$. The band splits as $J_{H}$ increases and both lattice geometries exhibit the same qualitative behavior. Since the hypercubic DOS is expected to have in-gap states, one may ask whether the metal-insulator transition is well defined for a half-filled band. We find that the band splitting in the Bethe and hypercubic lattices happens at the same critical value $\left(J_{H} S\right)_{c}=1 / \sqrt{2}$. At the transition, the imaginary part of the self-energy at $\omega=0$ vanishes and the real part diverges. As a result, $N(\omega=0)$ is exactly zero for $J_{H}>J_{H}^{c}$ and the metal-insulator transition for the half-filled band in the hypercubic geometry is well defined ${ }^{19}$ At small energies, $N(\omega) \propto e^{-\left(J_{H} S\right)^{4} / \omega^{2}}$ vanishes quite abruptly ${ }^{20}$ In contrast, the "outer" tails of the upper and lower bands behave very similar to the "bare" Gaussian form. Note that

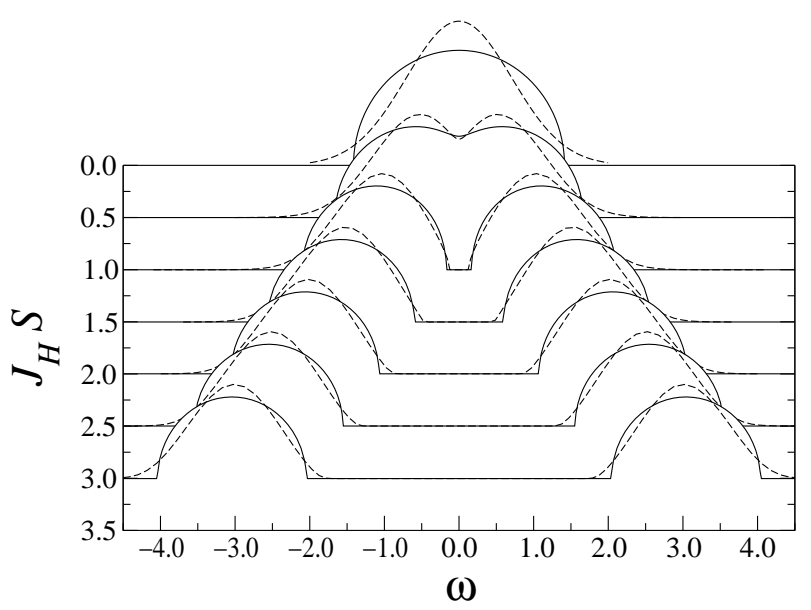

FIG. 1: Interacting DOS $N(\omega)$ for several values of $J_{H} S$ for the Bethe (solid lines) and hypercubic (dashed lines) lattices. 
for $J_{H} S \gtrsim 1$, the form of $N(\omega)$ for each subband is rather insensitive to the further increase of $J_{H}$.

We now rewrite Eq. (5) for the spin-diffusion coefficient within the DMFT:

$$
\frac{\mathcal{D}_{s} \chi}{a^{2}}=\frac{1}{2 z} \int_{-\infty}^{\infty} d \nu\left(-\left.\frac{\partial n(x)}{\partial x}\right|_{x=\nu}\right)\left[\frac{1}{b}-\frac{\partial}{\partial b}\right] \hat{A}(\nu),
$$

where $b=-\operatorname{Im} \Sigma(\nu)$ and $\hat{A}(\nu)=\int d \varepsilon \hat{\rho}_{0}^{\alpha}(\varepsilon) A_{\varepsilon}(\nu)$ with $\hat{\rho}_{0}^{\alpha}(\varepsilon)$ being the "current" DOS defined from the transformation $\sum_{\mathbf{k}} \sin ^{2} k_{i} a \Rightarrow \int d \varepsilon \hat{\rho}_{0}(\varepsilon) \stackrel{21}{=}$ For the hypercubic lattice $\hat{\rho}_{0}^{H}(\varepsilon)=\rho_{0}^{H}(\varepsilon) / 2$ while for the Bethe lattice $\hat{\rho}_{0}^{B}(\varepsilon)=\left(2-\varepsilon^{2}\right) \rho_{0}^{B}(\varepsilon) / 3$. To obtain Eq. (8) we used the relation $A_{\varepsilon}(\nu)^{2}=[1 / b-\partial / \partial b] A_{\varepsilon}(\nu) / 2 \pi$. Since the spin conductivity is proportional to the correlation function of two spin currents, each scaling as $t \sim 1 / \sqrt{z}$, the prefactor in Eq. (8) contains $1 / z$. This means that $d \mathcal{D}_{s} \chi$ is finite as $d \rightarrow \infty$, similar to the particle conductivity 22

In the manganites, the Curie temperature $T_{c}$ is much smaller than either the Hund's coupling or the bandwidth, in agreement with DMFT and Monte Carlo calculations for the DE model ${ }^{10.23}$ Therefore, all realistic temperatures are much smaller than the bandwidth $T \ll W$ and the derivative of the Fermi-function in the integrand of Eq. (8) should be replaced by a $\delta$-function at the chemical potential. Then, combining Eq. (8) with the specific form of the DOS's for the Bethe and hypercubic lattices, one arrives at:

$$
\frac{\mathcal{D}_{s} \chi z}{a^{2}}=\left\{\begin{array}{l}
\frac{1}{6 \pi}\left(2-\frac{g^{\prime \prime}}{b}\left(2-2 b^{2}-f^{2}\right)-f g^{\prime}\right)_{\nu=\mu}^{B} \\
\frac{1}{4 \pi}\left(2-\frac{g^{\prime \prime}}{b}\left(1-2 b^{2}\right)-2 f g^{\prime}\right)_{\nu=\mu}^{H},
\end{array},\right.
$$

where $g^{\prime}=\operatorname{Re} g(\nu), g^{\prime \prime}=\operatorname{Im} g(\nu)$, and $f=\nu-\operatorname{Re} \Sigma(\nu)$. Thus, the spin-diffusion coefficient is expressed through the local electronic Green's function and self-energy only. Fig. 2 presents $\mathcal{D}_{s} \chi$ as a function of the electronic concentration $n$ for several $J_{H} S$. As $n$ varies from 0 to 1 , the chemical potential sweeps from $\omega=-\infty$ to $\omega=0$ in Fig. 11 The results are very similar in both geometries and become independent of $J_{H} S$ as $J_{H} S \rightarrow \infty$ with the maximum located at $n=0.5$. In the limit $J_{H} S \gg 1$, Eq. (91) yields a numerical value for this maximum in $\mathcal{D}_{s} \chi z / a^{2}: 5 / 6 \pi(=0.265)$ and 0.292 for the Bethe and hypercubic case, respectively. This demonstrates a close quantitative correspondence between the results in the Bethe and hypercubic lattices, which justifies the use of the former despite the concerns outlined earlier. An interesting feature appears in the results for the hypercubic lattice as $n \rightarrow 0$. Instead of vanishing, the spin-diffusion coefficient tends to a finite limit $\mathcal{D}_{s} \chi z / a^{2}=1 / 4 \pi\left(J_{H} S\right)^{2}$, shown by circles in Fig. $2^{24}$

In the high-temperature limit $T \gg J_{H} S \gg 1$ Eq. (8) yields the result $\mathcal{D}_{s} \chi \sim 1 / T$ obtained previously using a Tchebycheff bounds (TB) formalism ${ }^{25}$ Numerically, $\left(\mathcal{D}_{s}^{D M F T} / \mathcal{D}_{s}^{T B}\right)_{\text {Bethe }}=40 \sqrt{2} / 9 \pi^{3 / 2}=1.13$ agree very closely as well.

It is interesting to analyze our results in the context of CMR materials. The superexchange (SE) interaction

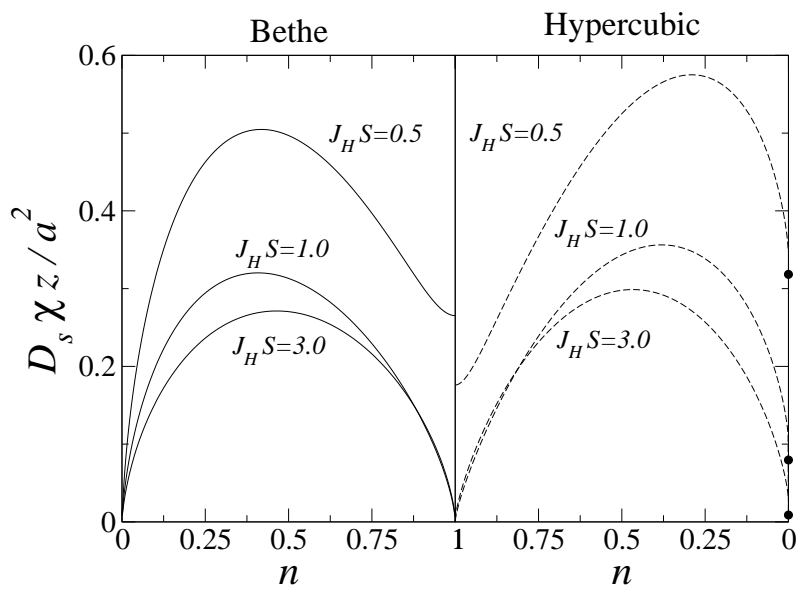

FIG. 2: $\mathcal{D}_{s} \chi z / a^{2}$ versus $n$ for several $J_{H} S$ for the Bethe (solid lines) and hypercubic (dashed lines) lattices.

is often discussed $\frac{5}{5}$ as necessary to correct the DE model. Within DMFT, the SE coupling $J^{S E}$ must scale as $1 / z$. Combining this with the result of Ref. 1 one finds that $\mathcal{D}_{s}^{S E} \propto 1 / z^{3 / 2}$ is suppressed in comparison with the DE result $\mathcal{D}_{s} \propto 1 / z$. This also serves as a demonstration that the DE model cannot be simply reduced to an effective Heisenberg model. In a real $d=3$ material the role of SE is further reduced by the smallness of $J_{i j}^{S E} \sim t^{2} / J_{H} S$ in comparison with the kinetic energy $\sim x t$. Therefore, the DE must dominate the spin diffusion and one can expect our results to be valid not only for the metallic part of the CMR phase diagram $\left(x=0.22 . .0 .5\right.$ for $\mathrm{La}_{1-x} \mathrm{Ca}_{x} \mathrm{O}_{3}$, $n=1-x$ in Fig. (2) but also for the ferromagnetic insulating phase $(x \leq 0.22) \stackrel{26}{\underline{n}}$ Of course, in the limit $x \rightarrow 0$ $\mathrm{DE}$ will diminish and the SE will dominate. Also, the critical scaling in the mixed phase $0<x<0.12$ (with a mixture of antiferromagnetic and ferromagnetic orders) can be strongly modified 27 These cases require separate consideration. At $x \geq 0.5$ charge ordering prevents the DE mechanism from being operative.

A systematic neutron scattering study of the metallic manganites recently focused on spin diffusion $\underline{3}^{\underline{3}}$ The width of the observed peak in $\mathcal{S}(\mathbf{q}, \omega)$ centered at $\omega=$ 0 scales as $\Lambda q^{2}$, where $\Lambda=2 \mathcal{D}_{s}$. Experimental results taken at $T^{\exp }(x) \simeq 1.1 T_{c}(x)$, where $T_{c}(x)$ is the Curie temperature for a given hole concentration $x$, give $\Lambda=15-30 \mathrm{meV}^{2}$. To compare our results to experiments we use $z=6$ and $a=3.87 \AA$. But keep in mind that spin diffusion persists below the transition point and that, at least for the experimentally accessible wave-vectors, the correlation length saturates at about 20 $\AA$. This implies that local magnetic correlations are suppressed by electronic inhomogeneities, which are probably associated with the local charge ordering $\underline{\underline{3}}$ So for simplicity, we take the susceptibility in the Curie form $\chi^{\exp }=S^{\prime}\left(S^{\prime}+1\right) / 3 T^{*}$, where $S^{\prime}(x)=S+(1-x) / 2$ is an average on-site spin and $T^{*}=T^{\exp }(x)$ is known from Ref. 3. Fig. [3] shows the theoretical data for $\mathcal{D}_{s} \chi / a^{2}$ for $J_{H} S \gg 1$ from Eq. (9) as a function of $x$ together with 


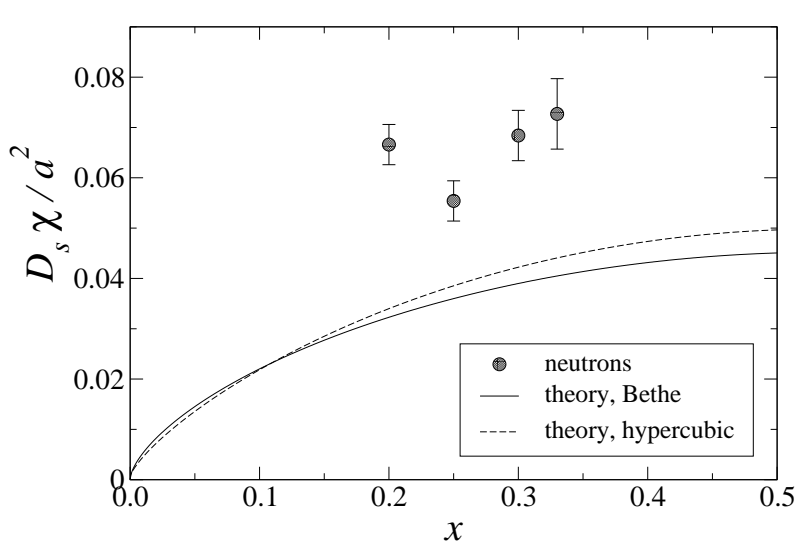

FIG. 3: $\mathcal{D}_{s} \chi / a^{2}$ as a function of $x$. Experimental values of $\mathcal{D}_{s}$ are from Fig. 5 of Ref. 3 , $\chi^{\exp }$ is described in the text, theoretical results are from Eq. (9) for $J_{H} S \gg 1$.

experimental $\mathcal{D}_{s}^{\exp } \chi^{\exp } / a^{2}$. We note here, that the theoretical curves in Fig. [3] are virtually independent of the actual value of $J_{H} S$ for $J_{H} S \gtrsim 1$. This figure demonstrates a remarkable agreement between the experimental and theoretical results, which contains no fitting parameters. Further improvement of the agreement can be sought, for example, from taking into account the second $e_{g}$ band which would effectively reduce $J_{H} / W \underline{\underline{13}}$
Since various other experiments also indicate the presence of local inhomogeneities in CMR systems, $\stackrel{28}{=}$ we propose the following analysis. As q decreases, long-range magnetic correlations must dominate $\chi(\mathbf{q})$ and the magnetic correlation length must eventually exceed the size of the local polaronic distortions. So at a fixed temperature close to $T_{c}, \chi^{\exp }(\mathbf{q})$ will increase and there will be a systematic decrease in the observed value of $\mathcal{D}_{s}^{e x p} \sim 1 / \chi$. This set of measurements would provide further information about magnetic correlations within the inhomogeneities in CMR systems.

In conclusion, we have presented a self-consistent study of the spin-diffusion in the double-exchange magnets within the framework of DMFT. This non-perturbative approach allows us to calculate the spin-diffusion coefficient at any temperature down to a transition point. A good agreement with the experiments in the ferromagnetic CMR manganites and earlier work is found. Altogether, this provides a new insight into the dynamics of strongly-correlated itinerant magnets.

We would like to acknowledge valuable discussions with A. H. Castro Neto, P. Dai, J. Fernandez-Baca, M. Jarrell, M. N. Kiselev, V. Perebeinos, and A. G. Yashenkin. This research was supported by ORNL, managed by UT-Battelle, LLC, for the U.S. DOE under contract DE-AC05-00OR22725.
1 P. Kopietz, Phys. Rev. B 57, 7829 (1998); Mod. Phys. Lett. B 7, 1747 (1993).

2 J. Bonca and J. Jaklic, Phys. Rev. B 51, 16083 (1995).

3 P. Dai et al., Phys. Rev. B 64, 224429 (2001).

4 M. E. Flatté and J. M. Byers, Phys. Rev. Lett. 84, 4220 (2000).

5 E. Dagotto et al., Phys. Rep. 344, 1 (2001) and Physics of Manganites, edited by T. A. Kaplan and S. D. Mahanti (Kluwer Academic and Plenum, New York, 1998).

6 D. Forster, Hydrodynamic Fluctuations, Broken Symmetry, and Correlation Functions, (Benjamin, Reading, 1975); B. I. Halperin and P. C. Hohenberg, Phys. Rev. 177, 952 (1969).

7 S. V. Maleev, Zh. Eksp. Teor. Fiz. 65, 1237 (1973) [Sov. Phys. JETP 38, 613 (1974)]; Yu. A. Izyumov and Yu. N. Skryabin, in Statistical Mechanics of Magnetically Ordered Systems, (Plenum, New York 1988).

8 G. D. Mahan, Many-Particle Physics, Third Edition (Kluwer Academic and Plenum, New York, 2000).

9 A. J. Millis et al., Phys. Rev. Lett. 77, 175 (1996).

10 N. Furukawa, J. Phys. Soc. Jpn. 64, 2754 (1995); 65, 1174 (1996).

11 D. I. Golosov, Phys. Rev. Lett. 84, 3974 (2000).

12 J. Zang et al., J. Phys.: Condens. Matter 9, L157 (1997).

13 A more realistic model with two $e_{g}$ bands becomes effectively equivalent to our single-band DE model Eq. (4) if the Jahn-Teller splitting or Hubbard repulsion are included. Thus, the two bands with splitting should only change our results quantitatively. Our theory can be extended to the Jahn-Teller case, but it transforms to a different problem for the case of the Hubbard interaction.
14 R. S. Fishman, Phys. Rev. B 62, R3600 (2000).

15 L.-J. Zou et al., J. Appl. Phys. 87, 5499 (2000).

16 A. Georges et al., Rev. Mod. Phys. 68, 13 (1996).

17 If the nano-inhomogeneities are truly important for $\mathrm{CMR}^{5}$ the DMFT will eventually fail to reproduce the percolative processes for transport in the CMR regime.

18 M. Auslender and E. Kogan, cond-mat/0102469

19 Also see A. O. Anokhin et al., J. Phys.: Condens. Matter 3, 1475 (1991).

20 Similar behavior has been found in the Hubbard model: A. Georges and W. Krauth, Phys. Rev. B 48, 7167 (1993).

21 A. Chattopadhyay et al., Phys. Rev. B 61, 10738 (2000).

${ }^{22}$ W. Metzner et al., Phys. Rev. B 45, 2237 (1992).

23 Y. Motome and N. Furukawa, J. Phys. Soc. Jpn. 69, 3785 (2000).

${ }^{24}$ In our problem, diffusion originates from correlations and the states in the remote tails $\left(|\mu| \gg J_{H} S\right)$ are weakly correlated with diverging relaxation time: $\tau \propto e^{\mu^{2}}$. The smallness of the DOS cancels this divergence giving a finite value of $\mathcal{D}_{s} \chi \propto \tau N(\mu)$ as $n \rightarrow 0$. In this limit the dynamical spindiffusion coefficient ${ }^{25} \mathcal{D}(\omega) \chi=\sigma_{s}(\omega) \propto 1 /\left(\omega^{2} \tau^{2}+4\right)$ has negligible width and weight given by $1 / \tau$.

25 R. S. Fishman, J. Phys.: Condens. Matter 12, L575 (2000); ibid. 14, 1337 (2002).

${ }^{26}$ In fact, the experimental data on $\mathcal{D}_{s}$ are very similar for the insulating and metallic compounds.$^{3}$

27 K. A. Kikoin and M. N. Kiselev, Zh. Eksp. Teor. Fiz. 112, 1816 (1997) [Sov. Phys. JETP 85, 994 (1997)].

28 C. P. Adams et al., Phys. Rev. Lett. 85, 3954 (2000). 